\title{
Correction to: Profiling Basal Forebrain Cholinergic Neurons Reveals a Molecular Basis for Vulnerability Within the Ts65Dn Model of Down Syndrome and Alzheimer's Disease
}

\author{
Melissa J. Alldred ${ }^{1,3}$. Sai C. Penikalapati ${ }^{1}$ - Sang Han Lee ${ }^{2} \cdot$ Adriana Heguy $^{4} \cdot$ Panos Roussos $^{1,5}$. \\ Stephen D. Ginsberg ${ }^{1,3,6,7}$
}

Published online: 27 November 2021

(c) Springer Science+Business Media, LLC, part of Springer Nature 2021

\section{Correction to: Molecular Neurobiology (2021) 58:5141-5162 https://doi.org/10.1007/s12035-021-02453-3}

The original version of this article unfortunately contained some mistakes.

The authors realized there are four citation errors in the Bioinformatics section of the Methods.

Citations 68-71 in the Methods erroneously link to citations in the Discussion. The actual 4 methods references were not cited.

With this, the correct citations and its bibliographical information are hereby given.

For the Bioinformatics Methods section:

EXISTING: "Fastqc version 0.11.8 [68]"

\#68 Wang X, Wang W, Li L, Perry G, Lee HG, Zhu X (2014) Oxidative stress and mitochondrial dysfunction in Alzheimer's disease. Biochim Biophys Acta 1842(8):1240-1247

The original article can be found online at https://doi.org/10.1007/ s12035-021-02453-3.

Stephen D. Ginsberg

ginsberg@nki.rfmh.org

Melissa J. Alldred

Melissa.Alldred@nki.rfmh.org

Sai C. Penikalapati

penikalapati.s@northeastern.edu

Sang Han Lee

SangHan.Lee@nki.rfmh.org

Adriana Heguy

Adriana.Heguy@nyulangone.org

Panos Roussos

Panagiotis.Roussos@nki.rfmh.org

1 Center for Dementia Research, Nathan Kline Institute, 140

Old Orangeburg Road, Orangeburg, NY 10962, USA
Corrected WITH: "Fastqc version 0.11.8 [130]"

130. Andrew S (2010) FastQC: A quality control tool for high throughput sequence data. https://www.bioinformatics. babraham.ac.uk/projects/fastqc/

EXISTING: "Trimmomatic 0.39 [69]."

\#69: Swerdlow RH (2018) Mitochondria and mitochondrial cascades in Alzheimer's disease. J Alzheimers Dis 62(3):1403-1416

Corrected WITH: "Trimmomatic 0.39 [131]."

131. Bolger AM, Lohse M, Usadel B (2014) Trimmomatic: a flexible trimmer for Illumina sequence data. Bioinformatics 30(15): 2114-2120

EXISTING: "using STAR Aligner (2.7.0) [70]."

\#70 Yamazaki Y, Zhao N, Caulfield TR, Liu CC, Bu G (2019) Apolipoprotein E and Alzheimer disease: pathobiology and targeting strategies. Nat Rev Neurol 15(9):501-518

2 Center for Biomedical Imaging and Neuromodulation, Nathan Kline Institute, Orangeburg, NY, USA

3 Departments of Psychiatry, New York University Grossman School of Medicine, New York, NY, USA

4 Genome Technology Center, New York University Grossman School of Medicine, New York, NY, USA

5 Departments of Genetics and Genomic Sciences and Psychiatry and the Institute for Data Science and Genomic Technology, Icahn School of Medicine at Mount Sinai, New York, NY, USA

6 Neuroscience \& Physiology, New York University Grossman School of Medicine, New York, NY, USA

7 NYU Neuroscience Institute, New York University Grossman School of Medicine, New York, NY, USA 
Corrected WITH: "using STAR Aligner (2.7.0) [132]."

132. Dobin AC, Davis CA, Schlesinger F, Drenkow J, Zaleski C, Jha P, et al (2013) STAR: ultrafast universal RNA-seq aligner. Bioinformatics 29:15-21

EXISTING: "Rseqc (v3.0.0) and Picard (2.20.03) [71]." \#71: Prendecki M, Florczak-Wyspianska J, Kowalska M, Ilkowski J, Grzelak T, Bialas K, Kozubski W, Dorszewska J (2019) APOE genetic variants and apoE, miR-107 and miR-650 levels in Alzheimer's disease. Folia Neuropathol 57(2):106-116

Corrected WITH: : "Rseqc (v3.0.0) and Picard (2.20.03) [133]."
133. Wang L, Wang S, Li W (2012) RSeQC: quality control of RNA-seq experiments. Bioinformatics 28(16):2184-2185

The online version of the original article can be found at https://doi.org/10.1007/s12035-021-02453-3.

Publisher's note Springer Nature remains neutral with regard to jurisdictional claims in published maps and institutional affiliations. 